\title{
RNA Is a Double-Edged Sword in ALS Pathogenesis
}

\author{
Benjamin L. Zaepfel ${ }^{1,2}$ and Jeffrey D. Rothstein ${ }^{3,4 *}$ \\ ${ }^{1}$ Biochemistry, Cellular and Molecular Biology Program, Johns Hopkins University School of Medicine, Baltimore, MD, \\ United States, ${ }^{2}$ Molecular Biology and Genetics Department, Johns Hopkins University School of Medicine, Baltimore, MD, \\ United States, ${ }^{3}$ Brain Science Institute, Johns Hopkins University School of Medicine, Baltimore, MD, United States, \\ ${ }^{4}$ Department of Neurology, Johns Hopkins University School of Medicine, Baltimore, MD, United States
}

OPEN ACCESS

Edited by:

Guey-Shin Wang,

Academia Sinica, Taiwan

Reviewed by:

Jun-An Chen,

Academia Sinica, Taiwan James N. Sleigh,

University College London, United Kingdom

*Correspondence:

Jeffrey $D$. Rothstein jrothst1@jh.edu

Specialty section: This article was submitted to

Cellular Neuropathology, a section of the journal

Frontiers in Cellular Neuroscience

Received: 11 May 2021

Accepted: 22 June 2021

Published: 19 July 2021

Citation:

Zaepfel BL and Rothstein JD (2021) RNA Is a Double-Edged Sword

in ALS Pathogenesis.

Front. Cell. Neurosci. 15:708181. doi: 10.3389/fncel.2021.708181
Amyotrophic lateral sclerosis (ALS) is a progressive and fatal neurodegenerative disease that affects upper and lower motor neurons. Familial ALS accounts for a small subset of cases $(<10-15 \%)$ and is caused by dominant mutations in one of more than 10 known genes. Multiple genes have been causally or pathologically linked to both ALS and frontotemporal dementia (FTD). Many of these genes encode RNA-binding proteins, so the role of dysregulated RNA metabolism in neurodegeneration is being actively investigated. In addition to defects in RNA metabolism, recent studies provide emerging evidence into how RNA itself can contribute to the degeneration of both motor and cortical neurons. In this review, we discuss the roles of altered RNA metabolism and RNA-mediated toxicity in the context of TARDBP, FUS, and C9ORF72 mutations. Specifically, we focus on recent studies that describe toxic RNA as the potential initiator of disease, disease-associated defects in specific RNA metabolism pathways, as well as how RNA-based approaches can be used as potential therapies. Altogether, we highlight the importance of RNA-based investigations into the molecular progression of ALS, as well as the need for RNA-dependent structural studies of disease-linked RNA-binding proteins to identify clear therapeutic targets.

Keywords: amyotrophic lateral sclerosis, TDP43, FTD, FUS, RNA, C9ORF72 ALS/FTD

\section{INTRODUCTION}

Since the discovery of mutations in TARDBP, FUS, and C9ORF72 that cause amyotrophic lateral sclerosis (ALS) and frontotemporal dementia (FTD), numerous investigations have demonstrated the effect these mutations have on RNA metabolism, as well as how RNA itself contributes to pathogenicity (Tollervey et al., 2011; Xiao et al., 2011; Daigle et al., 2013; Donnelly et al., 2013; Gendron et al., 2013; Ihara et al., 2013; Lagier-Tourenne et al., 2013; Lee et al., 2013; Nakaya et al., 2013; Shodai et al., 2013; Cooper-Knock et al., 2014; Coyne et al., 2014, 2015, 2017a, 2020; Highley et al., 2014; Liu-Yesucevitz et al., 2014; Mackness et al., 2014; Barmada et al., 2015; Burguete et al., 2015; Tran et al., 2015; Conlon et al., 2016; Dodd et al., 2016; Garnier et al., 2017; Liu et al., 2017; Lu et al., 2017; Kamelgarn et al., 2018; Tank et al., 2018; Chen et al., 2019; Xu et al., 2019; Ortega et al., 2020; Sun et al., 2020; Zaepfel et al., 2021). Mutations in all three of these genes have been linked to alterations in mRNA splicing and stress granule formation (Daigle et al., 2013; Nakaya et al., 2013; Conlon et al., 2016; Donde et al., 2019; Klim et al., 2019). However, we do not discuss such defects herein. Here, we discuss a bulk of the findings where direct or indirect alterations in RNA-related pathways have been linked to mutations in these three genes. We also address the role of RNA itself in mediating this toxicity. With a focus on the structure of proteins as they relate to RNA-binding 
and -processing functions, we provide new insight into the bi-directional effect between RNA itself and altered RNA processing in ALS.

\section{TDP-43}

\section{Introduction}

TARDBP encodes the TAR DNA-binding protein (TDP-43), which was initially discovered as a protein that binds pyrimidinerich regions (TAR) within the HIV-1 gene, repressing its expression (Ou et al., 1995). Novel RNA-binding domains were identified within TDP-43, which show remarkable affinity for UG repeat motifs (Buratti and Baralle, 2001). Although normally compartmentalized within the nucleus, TDP-43 was first implicated in neurodegeneration when it was found that its presence in ubiquitin-positive cytoplasmic inclusions is a hallmark of sporadic ALS and FTD (Arai et al., 2006; Neumann et al., 2006). The cytoplasmic shift of TDP-43 has also been linked to its nuclear clearance (Mitra et al., 2019; Nana et al., 2019), resulting in multi-faceted hypotheses for its sequential loss- and gain-of-function. While its nuclear clearance has most clearly been linked to loss of splicing regulation (Donde et al., 2019; Melamed et al., 2019), increased cytoplasmic abundance has been observed to alter multiple cytoplasmic pathways. These two pathologies have reliably been observed in some CNS cells in up to $97 \%$ of all sporadic ALS cases (Ling et al., 2013). Notably, although virtually all ALS cases have TDP43 aberrant pathology in CNS cells, only a small portion of neurons within each case demonstrate this pathology.

Soon after the observation of its pathology in sporadic ALS, coding mutations in TARDBP were shown to be causative of ALS in a rare number of dominantly inherited cases of familial ALS, introducing a complicated framework in which TDP-43 is both a pathological hallmark of sporadic ALS and functionally related to some, but not all familial causes of ALS pathogenesis (Kabashi et al., 2008; Sreedharan et al., 2008; Pesiridis et al., 2009). Since then, novel functions of TDP-43 in numerous aspects of RNA metabolism have been identified and linked to its involvement in ALS. The following section provides focused insight into how TDP-43 and RNA interact to mediate neuronal degeneration.

\section{Structure of TDP-43}

After the initial identification of RNA recognition motif (RRM) domains within TDP-43 (Buratti and Baralle, 2001), numerous attempts have been made to fully resolve the protein's fulllength structure. The low complexity domain of TDP-43 has significantly slowed, but not halted, progress on this front. The crystal structure of the TDP-43 RRM1 domain bound to DNA provided significant insight into its affinity for TG- and UG-rich sequences, especially when TDP-43 homodimerizes (Kuo et al., 2014), which was originally observed in previous characterizations of TDP-43 RNA targets (Tollervey et al., 2011; Xiao et al., 2011). This homodimerization depends upon intermolecular interactions between partially helical structure within the C-terminal of TDP-43 (Conicella et al., 2020). Dimerization is also dependent on the conserved E246 and D247 residues within the RRM2 domain (Shodai et al., 2012). The D247 residue is also part of a salt bridge between the two RRM domains with R151, which regulates RNA-binding function and specificity for TDP-43 (Flores et al., 2019).

Much is known about how specific point mutations confer changes to the function of TDP-43. Until recently, the lack of a high-yield protocol to produce pure, full-length TDP-43 has plagued the field. However, a major breakthrough by the Chiti group has made this possible (Vivoli Vega et al., 2019), opening the door for future investigations into the full structure and function of TDP-43 in its monomeric and homodimerized forms. Additionally, shortened isoforms of TDP-43 (sTDP-43) have been discovered in neurons that exhibit hyperactivity, further increasing the need for structural studies of full length and sTDP-43 (Weskamp et al., 2020).

\section{Role of TDP-43 and Its Mutant Variants in RNA Metabolism}

Since TDP-43 has roles in almost every aspect of RNA metabolism (Coyne et al., 2017b), it is no surprise that its diseaserelated mutations have been linked to dysfunction in numerous RNA pathways. One of the first indications that the RNAbinding function of TDP-43 is altered in neurological disease came from iCLIP-RNA Sequencing experiments performed on postmortem tissue samples of FTD patients (Tollervey et al., 2011; Xiao et al., 2011). These data support altered affinity of TDP-43 for certain RNA targets, ultimately leading to altered splicing function (Tollervey et al., 2011; Xiao et al., 2011).

TDP-43 mutations were soon linked to alterations in translation of target mRNAs. In a Drosophila model of ALS, overexpression of wild-type and mutant TDP demonstrated loss of actively translated futsch mRNA (MAP1B in humans) (Coyne et al., 2014). The direct binding of TDP-43 to futsch mRNA via a UG-rich region within its $5^{\prime}$ UTR was later validated (Romano et al., 2016). This reduced translation directly leads to reduced Futsch/Maplb protein, and the destabilization of neuromuscular junctions (Coyne et al., 2014). Furthermore, it was found that Drosophila FMRP (FMR1 in humans) can mitigate this translational deficit and remodel RNA granules to restore the translation of Futsch/Maplb to rescue synaptic defects (Coyne et al., 2015). The same model system was used to identify Hsc70-4 (HSPA8 in humans) as an additional translational target of TDP43 (Coyne et al., 2017a). The observed association between TDP43 and the ribosome protein RACK1 supports the possibility that TDP-43 mutations also have a much broader propensity to alter or reduce translation (Russo et al., 2017). An independent study observed no evidence of global translation defects caused by wildtype TDP-43 or TDP-43 A315T mutant, but did provide evidence that this mutant specifically increases translation of several target mRNAs (Camta1, Mig12, and Dennd4a) (Neelagandan et al., 2019; Figure 1).

There is also evidence that specific TDP-43 mutations or overexpression may lead to alterations in non-sense-mediated decay (NMD) (Barmada et al., 2015). NMD is a translationdependent RNA surveillance pathway that identifies and degrades mRNAs containing a premature termination codon or 


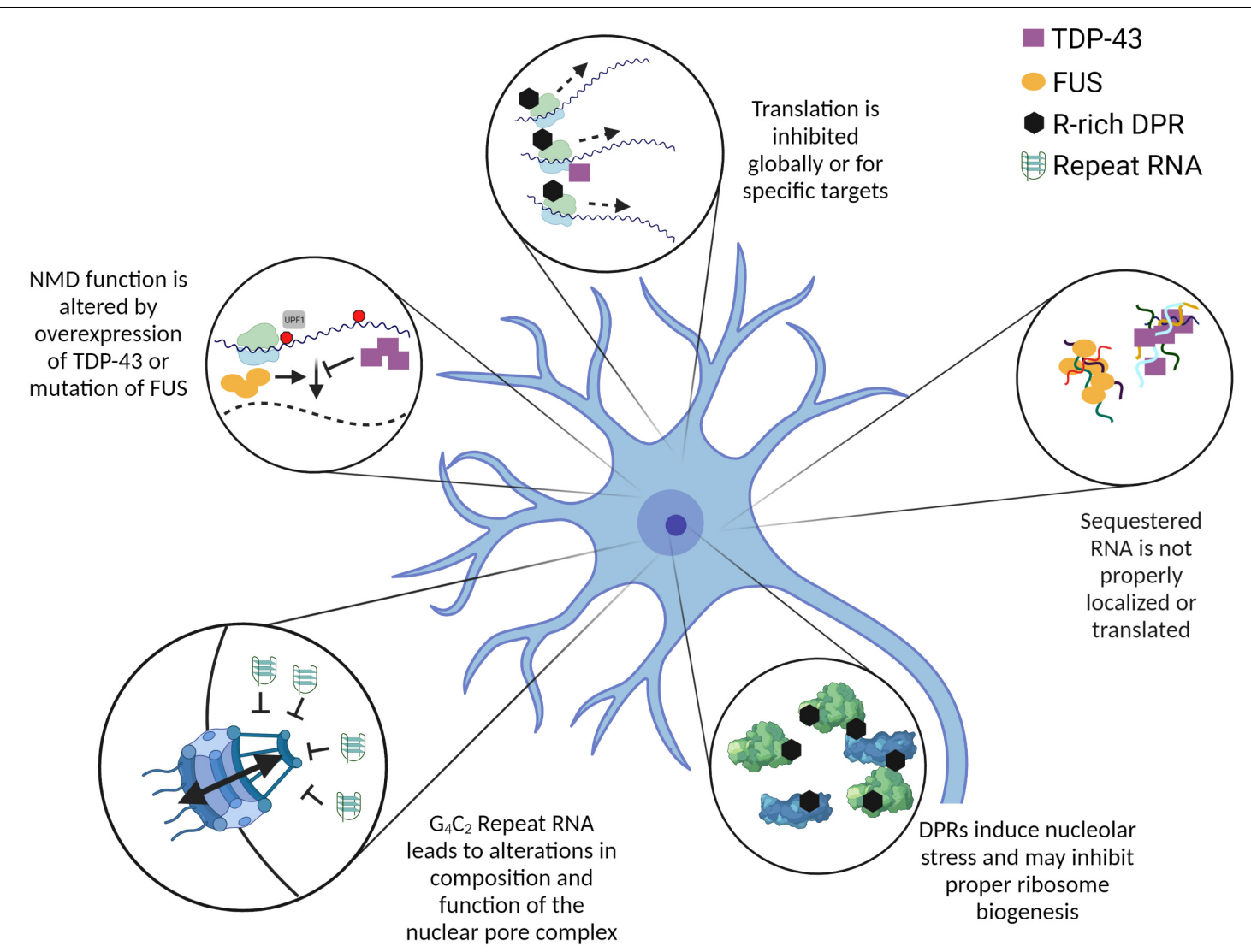

FIGURE 1 | Mutations and overexpression of ALS-related proteins and RNA contribute to altered RNA metabolism. The pathways and processes depicted here are altered by the overexpression or mutation of ALS-related genes. While the mechanisms leading to these alterations are not all fully characterized, it is evident that many RNA metabolic pathways are dysregulated in the context of ALS. In some cases, such as C9ALS, RNA itself can have a significant impact on cellular homeostasis. Further investigation into the mechanistic links between these altered processes will be crucial to understand the initiating event(s) in ALS pathogenesis. Created with BioRender.com.

particularly long 3' UTR (Kurosaki and Maquat, 2016). Notably, NMD requires an RNA-binding protein called UPF1 (LykkeAndersen et al., 2000). In mouse primary neurons overexpressing wild-type or mutant TDP-43, UPF1 overexpression improves survival, and this rescue is depends on NMD activity of UPF1 (Barmada et al., 2015). No direct analysis of NMD function was performed in this study. However, since TDP-43 may reduce translation globally, and NMD is a translation-dependent process, it is certainly possible that a primary translation defect could lead to an apparent secondary defect in the degradation of endogenous NMD substrates (Figure 1). This lurking variable necessitates future investigations into the link between TDP-43induced translation or splicing defects and observed alterations in downstream RNA metabolism.

Here, we only discuss RNA-binding in the context of cytoplasmic gain-of-function as it relates to TDP-43 and its disease-related mutants. However, there is powerful recent work that discusses the loss of nuclear TDP-43 function following its depletion from the nucleus (Highley et al., 2014; Donde et al., 2019; Klim et al., 2019; Melamed et al., 2019). It is important to note that while we discuss "RNA-binding" in a black-orwhite manner, the full biology of TDP-43 is laden with target- and compartment-specific functions that make broad statements about its RNA-binding quite enigmatic.

\section{Role of RNA in TDP-43-Mediated Toxicity}

In addition to the strong effect of TDP-43 on RNA metabolic processes in the cell, RNA has a robust effect on the concerted toxicity of TDP-43 in ALS (Voigt et al., 2010; Ihara et al., 2013; D’Alton et al., 2014; Kitamura et al., 2016; Chen et al., 2019). One of the first indications that RNA may mediate TDP-43 toxicity came from a targeted screen of TDP-43 variants in embryonic chick spinal cords. TDP-43 C-terminal fragment (CTF, which lacks most of RRM1 domain) and FF/LL (RNA-binding deficient) variants lead to significantly less neuronal death than WT or A315T TDP-43 when stably expressed unilaterally in Gallus gallus chicks (Voigt et al., 2010). An additional study in Drosophila demonstrated that while overexpression of WT TDP-43 in photoreceptors leads to significant cell death, removal of RRM1 or a pair of point mutations in RRM1 yield a clear reduction in toxicity (Ihara et al., 2013). Notably, this Drosophila model also shows that the considerable degeneration caused by $\triangle$ NLS-TDP43 is completely mitigated by removing the RRM1 domain (Ihara et al., 2013). Together, these investigations provide powerful 
in vivo evidence that the RNA-binding function of TDP-43 may be required to induce cellular toxicity.

One finding (at first glance) may seem to contradict this hypothesis of RNA-induced toxicity. The naturally occurring C-terminal cleavage product of TDP-43 (TDP-25) leads to significantly more cell death in Neuro2A cells when compared to TDP-43, yet TDP-25 does not colocalize with RNA in cells (Kitamura et al., 2016). Thus, it is important to note at this point that toxicity resulting from overexpression of various TDP43 species does not account for differences in their natural abundance. The C-terminal fragments of TDP-43 (TDP-25 and TDP-35) are significantly less abundant than the full-length TDP43 (D'Alton et al., 2014). While TDP-25 overexpression may lead to cellular toxicity without the need to bind RNA, this by no means diminishes this requirement for full-length TDP43 toxicity.

Domain-deletions provide powerful insight into the functions of TDP-43 that potentially lead to toxicity. Elucidating the neurodegenerating mechanism of ALS-causing mutations may be even stronger. Most TDP- 43 mutations are in the C-terminal domain and do not directly interfere with RNA processing or binding functions (Chen et al., 2019). One RRM1-adjacent mutation (K181E) provides key pieces of information: K181E cannot bind RNA and leads to reduced solubility and phosphorylation of itself and WT TDP-43 (Chen et al., 2019). As many studies have shown with overexpression and in vitro systems, solubility and phosphorylation of TDP-43 may be closely linked to its cytotoxicity (Arai et al., 2006; Neumann et al., 2006; Shodai et al., 2012, 2013; Sun et al., 2014; Coyne et al., 2015; Afroz et al., 2017; Li et al., 2017; Chen et al., 2019; French et al., 2019; Zacco et al., 2019). Although this review will not probe deeper into this side of TDP-43 biology, it is crucial to note that its RNA-binding function may inherently dictate its solubility.

\section{FUS}

The fused in sarcoma/translocated in liposarcoma (FUS) protein was first identified as part of a fusion protein resulting from a chromosomal translocation that is common in human myxoid liposarcomas (Crozat et al., 1993). Its clear role in cancer progression led to numerous studies to understand how FUS can act as a modulator of various disease phenotypes. Importantly, the Morikawa group characterized the structure of FUS as it relates to its RNA-binding properties (Iko et al., 2004). Four years later, mutations in FUS were found to be causative of familial ALS[69; 70]. Since then, investigations have been focused on how these mutations alter wild-type FUS function and lead to new toxic roles for FUS in motor neurons. The following section will discuss the role of FUS mutations in altered RNA metabolism, as well as how RNA itself might mediate the conferred toxicity.

\section{Structure of FUS}

Fused in sarcoma/translocated in liposarcoma is a ubiquitous RNA- and DNA-binding protein that is primarily localized to the nucleus (Crozat et al., 1993). It contains three arginine-glycineglycine (RGG) domains, with the first two separated by an RRM domain, and the last two separated by a zinc-finger domain (Crozat et al., 1993). Due to the intrinsic disorder of the RGG domains, a full crystal structure of FUS has not yet been resolved.

Recently, the solution structure of the RRM, second RGG domain (RGG2), and the zinc-finger ( $\mathrm{ZnF}$ ) domain (amino acids 269-454) were resolved in complex with various RNA motifs (Loughlin et al., 2019). This study provided novel insights into the specificity of these three RNA-binding domains for distinct RNA motifs and secondary structures. The RRM domain was observed to bind to a broad range of RNA motifs, including a stem-loop and single-stranded RNA (ssRNA) of varying sequences. The $\mathrm{ZnF}$ domain bound strongly to a UGGUA motif, while exhibiting very little binding to the other tested ssRNAs. Interestingly, no significant binding occurred with RGG2 for any of the RNA motifs tested (Loughlin et al., 2019).

In the context of ALS, it is notable that none of the FUS mutations identified in ALS patients are within the RRM or $\mathrm{ZnF}$ domains. One explanation is that mutations within these domains are physiologically silent while alternatively they may be lethal. FUS is non-essential in murine (Kino et al., 2015) and human cell (Reber et al., 2018) models. Thus, if mutations within these domains are lethal, it is unlikely that this lethality is due to loss of normal FUS function. Instead, it is possible that such mutations lead to aberrant (and lethal) function, perhaps via promiscuous RNA-binding. Indeed, a triplet of $\mathrm{K} \rightarrow \mathrm{A}$ mutations within the RRM lead to a loss of FUS- $\triangle$ NLS ability to compartmentalize itself within either the nucleus or cytoplasm (Liu et al., 2013). It is unknown how these mutations affect the function of full-length FUS.

As more complete structures of FUS become resolved, it will be crucial to review our current understanding of FUS structural mutations as they relate to ALS pathogenesis.

\section{Role of FUS Mutations in RNA Metabolism}

As would be expected of an RNA-binding protein, many of the ALS associated FUS mutations alter the protein's function within various RNA metabolism pathways. When mutations in FUS were first identified to cause ALS, the main observation involved these mutants' mislocalization to the cytoplasm (Kwiatkowski et al., 2009; Vance et al., 2009). Since then, a significant portion of the ALS-associated FUS mutations have been observed within the nuclear localization signal (NLS), residues 510-526 (Shang and Huang, 2016).

Fused in sarcoma/translocated in liposarcoma P525L has been shown to localize in large cytoplasmic accumulations when overexpressed in SH-SY5Y cells, and poly-A mRNA is enriched in these accumulations (Takanashi and Yamaguchi, 2014; Figure 1). However, the authors establish no causal link between this observation and potentially altered RNA metabolism. Theoretically, the mRNA present within these accumulations would be resistant to degradation by typical surveillance pathways. While this may shift the global transcriptome by stabilizing a subset of mRNA, it is unclear how this may functionally affect the proteome, as these mRNAs may not be translated while they are sequestered. 
Kinesin-1 (Kif5b) mRNA and protein are particularly enriched in FUS inclusions (Yasuda et al., 2017). This leads to a deficit in the ability of kinesin-1 to transport specific mRNAs to neuronal axons (Yasuda et al., 2017). Although the authors provide no experimental connection between mis-localization of kinesin-1 target mRNAs and known ALS pathogenesis, it is possible that hereunto unidentified mRNAs are similarly mislocalized, contributing directly to neuronal degeneration. Kinesin-1 also has a role in transporting materials anterogradely along axons (Lim et al., 2017), though it is not yet known whether FUS inclusions modulate this particular function of kinesin1. Interestingly, mutations in KIF5A, another gene encoding a kinesin protein, have recently been shown to cause ALS (Brenner et al., 2018; Nicolas et al., 2018). This warrants additional investigations into the possible connection between FUS and KIF5A in the context of ALS.

Whereas previous reports indicate reduced NMD activity in TDP-43 models of ALS (Barmada et al., 2015), FUS models hint at potentially increased NMD activity. Fibroblasts from patients with mutations in FUS have relatively high levels of UPF1 (Kamelgarn et al., 2018). This result, in addition to the reduced expression of several NMD reporters, is interpreted to indicate an increase in the activity of the NMD pathway. The authors present a model of mutant FUS-disrupted autoregulation within the NMD pathway (Kamelgarn et al., 2018; Figure 1). However, further investigation is needed to reconcile the apparent disconnect between the observed reduction in translation and increase in NMD activity caused by FUS mutations.

\section{Role of RNA in FUS-Mediated Toxicity}

Amyotrophic lateral sclerosis-linked FUS mutations clearly alter RNA metabolism, but this influence is not a one-way street. RNA itself can have a profound impact on FUS-mediated cellular toxicity. The study of FUS mutants with reduced or absent RNAbinding ability has provided a wealth of information about how RNA can mitigate, or instigate, toxicity.

Fused in sarcoma/translocated in liposarcoma has been shown to bind RNA via two different types of interaction: a monomer binding tightly to ssRNA, and a pair of monomers binding weakly (Niaki et al., 2020). This binding depends on the length of the RNA, and is significantly altered by mutations in FUS arginine residues (R244, R216, and R514), contributing to these mutants' propensity to form larger droplets than FUS WT (Niaki et al., 2020). Interestingly, the ubiquilin-like protein ubiquilin-2 is able to modify the FUS-RNA interaction, reducing its tendency to form large liquid droplets in vitro or stress granules in cells (Alexander et al., 2018). No direct assay was performed to determine if these larger droplets may reduce survival or lead to cellular toxicity. In vitro, RNA has the effect of reducing FUS liquid-liquid phase separation (LLPS) and helps maintain FUS solubility in the nucleus (Monahan et al., 2017; Maharana et al., 2018).

Expression of cytoplasmic FUS that lacks the RRM in mice leads to early death, although these mice don't exhibit signs of significant neurodegeneration, despite the presence of FUS inclusions in the cortex and brainstem (Robinson et al., 2015), and thus the actual mechanism underlying death is not known and could involve non-CNS tissues in this mouse model. This is not unprecedented, as early mutant TDP-43 mouse models died as a result of gastrointestinal problems, (not seen in the true human disease) rather than CNS degeneration (Hatzipetros et al., 2014).

While there is clear evidence in vitro that loss of the FUSRNA interaction can lead to formation of larger aggregates or inclusions, it is not fully clear that this has any neuronal-specific consequence in vivo. In order to truly understand whether FUSRNA binding is a significant contributor to ALS pathogenesis, it may be necessary to use in vivo knock-in studies to perform physiologically relevant experiments (Lopez-Erauskin et al., 2018; Reber et al., 2018).

\section{C9ORF72}

\section{Introduction}

Although many of the genes linked to ALS demonstrate diseasecausing point mutations, the C9ORF72 mutation exists as a GGGGCC hexanucleotide repeat expansion (HRE) within the first intron of the gene (DeJesus-Hernandez et al., 2011; Renton et al., 2011). This mutation accounts for the largest cohort of both familial (20-50\%) and sporadic (5-20\%) ALS (sALS) cases (collectively referred to as C9ALS) (Ling et al., 2013). In affected individuals, the HRE may be thousands of repeats long, but can also exhibit intermediate toxicity with as few as 24 repeats (Iacoangeli et al., 2019). Notably, this expansion ranges from 0 to 24 repeats in unaffected individuals. The HRE leads to three primary changes in neurons, each of which may contribute to the overall toxicity caused by the mutation.

First, the HRE reduces the abundance of C9ORF72 mRNA (DeJesus-Hernandez et al., 2011) and C9ORF72 protein (Renton et al., 2011; Waite et al., 2014). C9ORF72 encodes C9ORF72, a protein which helps regulate autophagy (Webster et al., 2018). Therefore, it has been hypothesized that haploinsufficiency of C9ORF72 reduces autophagic regulation. Notably, heterozygous loss of the protein/mRNA, comparable to human disease has no overt pathological toxicity, although subtle changes in microglial RNA have been detected. In that regard, an increase in autoimmune diseases have been seen a very small number of C9ALS/FTD patients-but to emphasize, C9ALS is not a disorder with prominent autoimmune disease burden. However, complete loss of the protein/RNA, not seen in human C9ALS, leads to a severe autoimmune dysfunction in rodent, not typical of C9ALS, or ALS in general (Ciura et al., 2013; Shi et al., 2018).

Second, the HRE-containing RNA, though present within an intron, translocates to the cytoplasm (Donnelly et al., 2013; Lagier-Tourenne et al., 2013). Here, it may aberrantly sequester miscellaneous proteins, diminishing their ability to function normally. Notably, the antisense repeat RNA (CCCCGG) may also be transcribed and translocated to the cytoplasm (Gendron et al., 2013).

Third, cytoplasmic repeat RNA can assume highly-structured forms (Fratta et al., 2012) which can recruit ribosomal machinery to initiate translation. Via this repeat-associated non-ATG (RAN) translation, ribosomes bind to the sense and antisense repeat 
RNA and elongate to produce dipeptide repeat (DPR) proteins[5; 96]. Owing to ribosomal frameshifting, two unique DPRs are produced from each HRE RNA strand, with a common DPR expressed by both [GGGGCC RNA yields poly(GA) and poly(GR), CCCCGG RNA produces poly(PA) and poly(PR), both strands yield poly(GP)] (Ash et al., 2013; Gendron et al., 2013; Zu et al., 2013; Tabet et al., 2018). These five DPRs have been hypothesized to induce toxicity through a plethora of mechanisms.

Due to the complex nature of this mutation, as well as the focus of this review, the following discussion will be limited to an assessment of how the HRE RNAs and DPRs alter RNA processing pathways, as well as how the repeat RNA itself induces neuronal toxicity.

\section{Structure of C9ORF72 HRE RNA and DPRs}

The GGGGCC HRE RNA has been shown to form G-quadruplexes in vitro (Fratta et al., 2012; Reddy et al., 2013; Conlon et al., 2016), and these structures have been observed in the postmortem tissue of ALS patients (Conlon et al., 2016). Few studies have delved into the structure of purified (GGGGCC) n RNA under various conditions (Dodd et al., 2016; Mulholland et al., 2020), though the physiological structure of this RNA remains elusive. It is known that some subset of the HRE RNA is present in the form of foci, which each contain a single highly structured RNA molecule (Liu et al., 2017), though studies of the structures that soluble HRE RNA adopts have yet to be performed. There remains an unmet need to understand the various states in which the HRE RNA exists in vivo. Future investigations may need to probe the distinct roles of soluble (unstructured) and insoluble (highly structured) HRE RNA in conferring cellular toxicity.

Due to the variable length of the five DPRs, as well as their relative insolubility when purified, it has been difficult for investigators to derive their crystal or nuclear magnetic resonance structures. However, some headway has been made using techniques like circular dichroism to elucidate the relatively disordered secondary structure of the DPRs (Flores et al., 2016). To date the relative amounts of the individual repeat polypeptides and the true range of their lengths in vivo remain unknown.

Clearly, the field of investigating the HRE RNA and DPR structures is wide open. Advancements therein will provide extremely useful data to inform future investigations into their toxic role in disease.

\section{Role of the C90RF72 Mutation in RNA Metabolism}

The first indication that the HRE RNA may contribute to alterations in RNA metabolism came with the observations that the HRE foci (punctate inclusions containing the HRE sense and antisense RNA) sequester various RNA-binding proteins (RBPs) (Donnelly et al., 2013; Lee et al., 2013; Cooper-Knock et al., 2014; Conlon et al., 2016). The hypothesis presented is that "locking up" of RBPs reduces their endogenous functions to maintain RNA homeostasis; this is a common feature among selected neurological/neuromuscular diseases having been previously well-studied in myotonic dystrophy (Jiang et al., 2004).

Dipeptide repeats have also been shown to interact with RBPs, altering their endogenous functions. Co-immunoprecipitation of synthetic poly(PR) after incubation with NSC34 cells yielded a variety of poly(PR)-interacting proteins that regulate translation and ribosome assembly (Kanekura et al., 2016). Independent screens also identify RBPs that are significantly altered in function by the presence of high poly(PR) and poly(GR) concentrations (Hartmann et al., 2018; Kramer et al., 2018; Zhang et al., 2018). These findings are strongly validated with the observation that the presence of high poly(PR) concentrations significantly reduced association of the crucial translation initiation factors eIF4E and eIF4G with poly(A) RNA (Kanekura et al., 2016). Additional experiments show that high poly(PR) or poly(GR) concentrations (via overexpression or exogenous addition) reduce translation (Hartmann et al., 2018; Zhang et al., 2018; Sun et al., 2020). Indeed, controlled titration of synthetic poly(PR $)_{20}$ shows almost perfect alignment with cycloheximide in terms of the effect on reducing translation (Sun et al., 2020; Figure 1).

The potent effect of $\operatorname{poly}(\mathrm{GR})$ and $\operatorname{poly}(\mathrm{PR})$ on translation becomes quite relevant with the "apparent" discovery of NMD defects in C9ALS. Multiple recent studies have proposed that NMD function is altered by the HRE, whether it be due to DPR toxicity or HRE RNA (Xu et al., 2019; Ortega et al., 2020; Sun et al., 2020). Notably, the observations of NMD dysfunction provided by these studies are either unconvincing (derived from minor phenotypes with a very low sample size) (Ortega et al., 2020) or stem from significant translation defects induced by overexpression of arginine-rich DPRs (Xu et al., 2019; Sun et al., 2020). Thus, it is difficult to conclude from these studies whether NMD function is at all altered by the HRE in human patients. A very new study has attempted to answer this question using an human induced pluripotent stem cell-derived spinal neuron (iPSN) model of C9ALS (Zaepfel et al., 2021). Following validation of several bona fide transcripts as substrates for NMD, it was demonstrated that neither the steady-state abundance nor stability of these substrates is altered in C9ALS patient-derived iPSNs relative to age- and sex-matched controls (Zaepfel et al., 2021). Although this strongly refutes the presence of any NMD dysfunction in C9ALS, this study corroborates that UPF1 is neuroprotective in this context (Zaepfel et al., 2021). Evidence is provided that UPF1 overexpression reduces the abundance of DPRs in multiple models, potentially explaining its neuroprotective effects (Zaepfel et al., 2021). However, much is still left unanswered by these papers to precisely explain the role that UPF1 plays in supporting neuronal health.

In addition to the strong RBP sequestration caused by the HRE RNA and DPRs, there is also evidence that the biogenesis of ribosomes may be disrupted by this mutation. Poly(GR) and poly(PR) colocalize with ribosomal proteins in C9ALS patient tissue (Hartmann et al., 2018), which hints at a possible unbalancing of the carefully maintained stoichiometry of ribosomal proteins with ribosomal RNA. When overexpressed, poly(PR) and poly(GR) both flock to nucleoli, the ribosomegenerating centers of the cell (Tao et al., 2015). This localization of arginine-rich DPRs to the nucleoli leads to nucleolar stress 
(Tao et al., 2015; Mizielinska et al., 2017; Hartmann et al., 2018), which may lead to significant alterations in the proper assembly of ribosomes themselves (Yang et al., 2018). There exists a possibility that this nucleolar stress leads to misassembly of ribosomes, which may compound the reduced translation noted above.

\section{Role of RNA in C9ORF72 HRE-Mediated Toxicity}

Relative to the plethora of investigations designed to understand the contribution of DPRs to neuronal toxicity, little is known about if and how the HRE RNA itself leads to disease phenotypes. This is due, in large part, to early Drosophila models that overexpress the HRE RNA or GGGGCC RNA that contains intermittent stop codons ("RNA only") (Mizielinska et al., 2014). While the former demonstrates production of both the repeat RNA and DPRs, RNA only flies produce only the RNA. These models, taken at face value, provided a controlled opportunity to determine the respective contributions of these two sets of toxic products. Indeed, severe degenerative phenotypes were observed in flies expressing the uninterrupted repeat, but flies expressing only the repeat RNA showed no signs of toxicity, despite containing repeat RNA foci (Mizielinska et al., 2014). This observation quite resoundingly turned most of the ALS field away from investigations of potential RNA-contributed toxicity. To many, elucidating a role for repeat RNA in HRE-mediated degeneration seemed like a proverbial search for a needle in a haystack without knowing that the needle exists.

This needle was recently found during a journey to understand the initiating event for nucleocytoplasmic transport dysfunction in C9ALS. Many labs have reported that neurons harboring the HRE demonstrate reduced ability to regulate transport across the nuclear pore complex (Freibaum et al., 2015; Zhang et al., 2015). For many years, little to no headway was made in identifying the root cause of this dysfunction. Then a comprehensive investigation identified the precise nucleoporins (the proteins that assemble into the nuclear pore complex) that are absent in C9ALSpatient neurons (Coyne et al., 2020). One such nucleoporin, Pom121, is proposed as the initial domino in a series of lost nucleoporins (Coyne et al., 2020). A painstaking number of orthogonal hypothesiskilling experiments brings the authors to a unified conclusion: the entirety of nuclear pore complex disassembly is instigated by the HRE RNA, not DPRs or loss of C9ORF72 protein (Coyne et al., 2020; Figure 1). The precise mechanism of HRE RNA-mediated disassembly of the nuclear pore complex remains enigmatic. Why, then, did that original Drosophila model fail to demonstrate RNA-induced toxicity? The answer is simple: Pom121 is not conserved in fruit flies (Coyne et al., 2020). Without the presence of this initial domino, the rest do not fall.

\section{RNA-Based Therapies for ALS}

RNA-centric therapies for ALS are quite varied, due to the distinct roles that RNA plays in instigating or ameliorating toxicity in different models as described above. In the case of
C9ALS, antisense oligonucleotides (ASOs) have been utilized widely in the laboratory and clinical settings. ASOs are heavily modified DNA sequences that are designed complimentary to a target RNA. Upon annealing, they induce RNase $\mathrm{H}$-dependent degradation of their target while the ASOs themselves remain intact (Ly and Miller, 2018). ASOs against the C9ORF72 HRE RNA, though initially developed as a tool to eliminate RNA foci in cultured cells (Donnelly et al., 2013; LagierTourenne et al., 2013), have been developed for trials in ALS patients, although the results of this novel intervention are as yet unknown. Since these initial ASOs targeted the sense strand repeat-containing introns of C9ORF72 RNA, the prediction is that pathogenic RNA will be degraded without affecting the abundance of coding mRNA. Whether ASO targeting the antisense strand may also be necessary for disease mitigation is unknown-but should be investigated at least preclinically.

Targeted knockdown of TDP-43 and FUS, however, may not be as useful. While it is a valid hypothesis that reducing their abundance would decrease their propensity for pathogenesis, both TDP-43 and FUS have important biological functions. Knocking down these mRNAs may open a whole new can of worms, where loss of their endogenous function may be just as toxic as their disease-related gain of function. Numerous in vitro and in vivo studies have clearly demonstrated that simple knockdown or overexpression of wild type TDP-43 is terribly cytotoxic. One workaround that may prove effective in the realm of TDP-43 pathogenesis comes with the use of bait oligonucleotides (Mann et al., 2019). Introduction of TDP43-targeted RNA oligonucleotides to cells overexpressing TDP43 leads to decreased neurotoxicity, hypothetically by reducing the aberrant phase transition of TDP-43 (Mann et al., 2019). Although this study erroneously counts individual cells as independent samples, the effect of this method of treatment is notable (Mann et al., 2019).

Targeting downstream molecular events that follow TDP-43 loss of function, such as Stathmin2 mis-splicing, are open to candidate therapies and may allow at least some mitigation of TDP-43 loss-of-function toxicity (Melamed et al., 2019). Finally, understanding the mechanism that lies upstream of aberrant TDP-43 loss of nuclear localization may prove to be the most encompassing approach to therapy - not only for ALS-but the other disorders of TDP-43 pathology.

\section{CONCLUSION}

Decades of research and independent investigations have provided clear evidence that RNA biology is commonly disrupted in the context of ALS. Yet our understanding of RNA metabolism alterations caused by each mutation lacks a unifying link. Indeed, the missing link may be found by investigating RNA metabolism in sALS, where the vast majority of cases are caused by hitherto unknown mechanisms. If a prominent, causative mechanism for sALS pathogenesis is identified within the realm of RNA biology, only then can the field clarify the bidirectional role for RNA in ALS neurotoxicity and neuroprotection. 


\section{AUTHOR CONTRIBUTIONS}

BLZ wrote the manuscript. JDR edited the manuscript. Both authors contributed to the article and approved the submitted version.

\section{REFERENCES}

Afroz, T., Hock, E. M., Ernst, P., Foglieni, C., Jambeau, M., Gilhespy, L. A. B., et al. (2017). Functional and dynamic polymerization of the ALS-linked protein TDP-43 antagonizes its pathologic aggregation. Nat. Commun. 8:45.

Alexander, E. J., Ghanbari Niaki, A., Zhang, T., Sarkar, J., Liu, Y., Nirujogi, R. S., et al. (2018). Ubiquilin 2 modulates ALS/FTD-linked FUS-RNA complex dynamics and stress granule formation. Proc. Natl. Acad. Sci. U.S.A. 115, E11485-E11494.

Arai, T., Hasegawa, M., Akiyama, H., Ikeda, K., Nonaka, T., Mori, H., et al. (2006). TDP-43 is a component of ubiquitin-positive tau-negative inclusions in frontotemporal lobar degeneration and amyotrophic lateral sclerosis. Biochem. Biophys. Res. Commun. 351, 602-611. doi: 10.1016/j.bbrc.2006.10.093

Ash, P. E., Bieniek, K. F., Gendron, T. F., Caulfield, T., Lin, W. L., DejesusHernandez, M., et al. (2013). Unconventional translation of C9ORF72 GGGGCC expansion generates insoluble polypeptides specific to c9FTD/ALS. Neuron 77, 639-646. doi: 10.1016/j.neuron.2013.02.004

Barmada, S. J., Ju, S., Arjun, A., Batarse, A., Archbold, H. C., Peisach, D., et al. (2015). Amelioration of toxicity in neuronal models of amyotrophic lateral sclerosis by hUPF1. Proc. Natl. Acad. Sci. U.S.A. 112, 7821-7826. doi: 10.1073/ pnas. 1509744112

Brenner, D., Yilmaz, R., Muller, K., Grehl, T., Petri, S., Meyer, T., et al. (2018). German, Hot-spot KIF5A mutations cause familial ALS. Brain 141, 688-697. doi: 10.1093/brain/awx370

Buratti, E., and Baralle, F. E. (2001). Characterization and functional implications of the RNA binding properties of nuclear factor TDP-43, a novel splicing regulator of CFTR exon 9. J. Biol. Chem. 276, 36337-36343. doi: 10.1074/jbc. m104236200

Burguete, A. S., Almeida, S., Gao, F. B., Kalb, R., Akins, M. R., and Bonini, N. M. (2015). GGGGCC microsatellite RNA is neuritically localized, induces branching defects, and perturbs transport granule function. eLife 4:e08881.

Chen, H. J., Topp, S. D., Hui, H. S., Zacco, E., Katarya, M., McLoughlin, C., et al. (2019). RRM adjacent TARDBP mutations disrupt RNA binding and enhance TDP-43 proteinopathy. Brain 142, 3753-3770. doi: 10.1093/brain/awz313

Ciura, S., Lattante, S., Le Ber, I., Latouche, M., Tostivint, H., Brice, A., et al. (2013). Loss of function of C9orf72 causes motor deficits in a zebrafish model of amyotrophic lateral sclerosis. Ann. Neurol. 74, 180-187.

Conicella, A. E., Dignon, G. L., Zerze, G. H., Schmidt, H. B., D’Ordine, A. M., Kim, Y. C., et al. (2020). TDP-43 alpha-helical structure tunes liquid-liquid phase separation and function. Proc. Natl. Acad. Sci. U.S.A. 117, 5883-5894. doi: 10.1073/pnas.1912055117

Conlon, E. G., Lu, L., Sharma, A., Yamazaki, T., Tang, T., Shneider, N. A., et al. (2016). The C9ORF72 GGGGCC expansion forms RNA G-quadruplex inclusions and sequesters hnRNP $\mathrm{H}$ to disrupt splicing in ALS brains. eLife 5:e17820.

Cooper-Knock, J., Walsh, M. J., Higginbottom, A., Robin Highley, J., Dickman, M. J., Edbauer, D., et al. (2014). Sequestration of multiple RNA recognition motif-containing proteins by C9orf72 repeat expansions. Brain 137, 2040-2051. doi: 10.1093/brain/awu120

Coyne, A. N., Lorenzini, I., Chou, C. C., Torvund, M., Rogers, R. S., Starr, A., et al. (2017a). Post-transcriptional Inhibition of Hsc70-4/HSPA8 expression leads to synaptic vesicle cycling defects in multiple models of ALS. Cell Rep. 21, 110-125. doi: 10.1016/j.celrep.2017.09.028

Coyne, A. N., Siddegowda, B. B., Estes, P. S., Johannesmeyer, J., Kovalik, T., Daniel, S. G., et al. (2014). Futsch/MAP1B mRNA is a translational target of TDP-43 and is neuroprotective in a Drosophila model of amyotrophic lateral sclerosis. J. Neurosci. 34, 15962-15974. doi: 10.1523/jneurosci.2526-14.2014

Coyne, A. N., Yamada, S. B., Siddegowda, B. B., Estes, P. S., Zaepfel, B. L., Johannesmeyer, J. S., et al. (2015). Fragile X protein mitigates TDP-43 toxicity

\section{FUNDING}

This work was supported by the NIH-NINDS, Department of Defense, F Prime, and the Chan Zuckerberg Initiative. Training grant T32GM007445 to BCMB.

by remodeling RNA granules and restoring translation. Hum. Mol. Genet. 24, 6886-6898.

Coyne, A. N., Zaepfel, B. L., Hayes, L., Fitchman, B., Salzberg, Y., Luo, E. C., et al. (2020). G4C2 Repeat RNA Initiates a POM121-mediated reduction in specific nucleoporins in C9orf72 ALS/FTD. Neuron 107, 1124-1140.e11.

Coyne, A. N., Zaepfel, B. L., and Zarnescu, D. C. (2017b). Failure to deliver and translate-new insights into RNA Dysregulation in ALS. Front. Cell Neurosci. 11:243. doi: 10.3389/fncel.2017.00243

Crozat, A., Aman, P., Mandahl, N., and Ron, D. (1993). Fusion of CHOP to a novel RNA-binding protein in human myxoid liposarcoma. Nature 363, 640-644. doi: $10.1038 / 363640 \mathrm{a} 0$

Daigle, J. G., Lanson, N. A. Jr., Smith, R. B., Casci, I., Maltare, A., Monaghan, J., et al. (2013). RNA-binding ability of FUS regulates neurodegeneration, cytoplasmic mislocalization and incorporation into stress granules associated with FUS carrying ALS-linked mutations. Hum. Mol. Genet. 22, 1193-1205. doi: $10.1093 / \mathrm{hmg} / \mathrm{dds} 526$

D’Alton, S., Altshuler, M., Cannon, A., Dickson, D. W., Petrucelli, L., and Lewis, J. (2014). Divergent phenotypes in mutant TDP-43 transgenic mice highlight potential confounds in TDP-43 transgenic modeling. PLoS One 9:e86513. doi: 10.1371/journal.pone.0086513

DeJesus-Hernandez, M., Mackenzie, I. R., Boeve, B. F., Boxer, A. L., Baker, M., Rutherford, N. J., et al. (2011). Expanded GGGGCC hexanucleotide repeat in noncoding region of C9ORF72 causes chromosome 9p-linked FTD and ALS. Neuron 72, 245-256. doi: 10.1016/j.neuron.2011.09.011

Dodd, D. W., Tomchick, D. R., Corey, D. R., and Gagnon, K. T. (2016). Pathogenic C9ORF72 antisense repeat RNA forms a double Helix with Tandem C:C mismatches. Biochemistry 55, 1283-1286. doi: 10.1021/acs.biochem.6b00136

Donde, A., Sun, M., Ling, J. P., Braunstein, K. E., Pang, B., Wen, X., et al. (2019). Splicing repression is a major function of TDP-43 in motor neurons. Acta Neuropathol. 138, 813-826. doi: 10.1007/s00401-019-02042-8

Donnelly, C. J., Zhang, P. W., Pham, J. T., Haeusler, A. R., Mistry, N. A., Vidensky, S., et al. (2013). RNA toxicity from the ALS/FTD C9ORF72 expansion is mitigated by antisense intervention. Neuron 80, 415-428. doi: 10.1016/j. neuron.2013.10.015

Flores, B. N., Dulchavsky, M. E., Krans, A., Sawaya, M. R., Paulson, H. L., Todd, P. K., et al. (2016). Distinct C9orf72-associated dipeptide repeat structures correlate with neuronal toxicity. PLoS One 11:e0165084. doi: 10.1371/journal. pone. 0165084

Flores, B. N., Li, X., Malik, A. M., Martinez, J., Beg, A. A., and Barmada, S. J. (2019). An intramolecular salt bridge linking tdp43 rna binding, protein stability, and TDP43-dependent neurodegeneration. Cell Rep. 27, 1133-1150 e8.

Fratta, P., Mizielinska, S., Nicoll, A. J., Zloh, M., Fisher, E. M., Parkinson, G., et al. (2012). C9orf72 hexanucleotide repeat associated with amyotrophic lateral sclerosis and frontotemporal dementia forms RNA G-quadruplexes. Sci. Rep. 2:1016.

Freibaum, B. D., Lu, Y., Lopez-Gonzalez, R., Kim, N. C., Almeida, S., Lee, K. H., et al. (2015). GGGGCC repeat expansion in C9orf72 compromises nucleocytoplasmic transport. Nature 525, 129-133. doi: $10.1038 /$ nature 14974

French, R. L., Grese, Z. R., Aligireddy, H., Dhavale, D. D., Reeb, A. N., Kedia, N., et al. (2019). Detection of TAR DNA-binding protein 43 (TDP-43) oligomers as initial intermediate species during aggregate formation. J. Biol. Chem. 294, 6696-6709. doi: 10.1074/jbc.ra118.005889

Garnier, C., Devred, F., Byrne, D., Puppo, R., Roman, A. Y., Malesinski, S., et al. (2017). Zinc binding to RNA recognition motif of TDP-43 induces the formation of amyloid-like aggregates. Sci. Rep. 7:6812.

Gendron, T. F., Bieniek, K. F., Zhang, Y. J., Jansen-West, K., Ash, P. E., Caulfield, T., et al. (2013). Antisense transcripts of the expanded C9ORF72 hexanucleotide repeat form nuclear RNA foci and undergo repeat-associated 
non-ATG translation in c9FTD/ALS. Acta Neuropathol. 126, 829-844. doi: 10.1007/s00401-013-1192-8

Hartmann, H., Hornburg, D., Czuppa, M., Bader, J., Michaelsen, M., Farny, D., et al. (2018). Proteomics and C9orf72 neuropathology identify ribosomes as poly-GR/PR interactors driving toxicity. Life Sci. Alliance 1:e201800070. doi: 10.26508/lsa.201800070

Hatzipetros, T., Bogdanik, L. P., Tassinari, V. R., Kidd, J. D., Moreno, A. J., Davis, C., et al. (2014). C57BL/6J congenic Prp-TDP43A315T mice develop progressive neurodegeneration in the myenteric plexus of the colon without exhibiting key features of ALS. Brain Res. 1584, 59-72. doi: 10.1016/j.brainres. 2013.10.013

Highley, J. R., Kirby, J., Jansweijer, J. A., Webb, P. S., Hewamadduma, C. A., Heath, P. R., et al. (2014). Loss of nuclear TDP-43 in amyotrophic lateral sclerosis (ALS) causes altered expression of splicing machinery and widespread dysregulation of RNA splicing in motor neurones. Neuropathol. Appl. Neurobiol. 40, 670-685. doi: $10.1111 /$ nan. 12148

Iacoangeli, A., Al Khleifat, A., Jones, A. R., Sproviero, W., Shatunov, A., OpieMartin, S., et al. (2019). C9orf72 intermediate expansions of 24-30 repeats are associated with ALS. Acta Neuropathol. Commun. 7:115.

Ihara, R., Matsukawa, K., Nagata, Y., Kunugi, H., Tsuji, S., Chihara, T., et al. (2013). RNA binding mediates neurotoxicity in the transgenic Drosophila model of TDP-43 proteinopathy. Hum. Mol. Genet. 22, 4474-4484. doi: 10.1093/hmg/ ddt296

Iko, Y., Kodama, T. S., Kasai, N., Oyama, T., Morita, E. H., Muto, T., et al. (2004). Domain architectures and characterization of an RNA-binding protein, TLS. J. Biol. Chem. 279, 44834-44840. doi: 10.1074/jbc.m408552200

Jiang, H., Mankodi, A., Swanson, M. S., Moxley, R. T., and Thornton, C. A. (2004). Myotonic dystrophy type 1 is associated with nuclear foci of mutant RNA, sequestration of muscleblind proteins and deregulated alternative splicing in neurons. Hum. Mol. Genet. 13, 3079-3088. doi: 10.1093/hmg/ddh327

Kabashi, E., Valdmanis, P. N., Dion, P., Spiegelman, D., McConkey, B. J., Vande Velde, C., et al. (2008). TARDBP mutations in individuals with sporadic and familial amyotrophic lateral sclerosis. Nat. Genet. 40, 572-574. doi: $10.1038 /$ ng. 132

Kamelgarn, M., Chen, J., Kuang, L., Jin, H., Kasarskis, E. J., and Zhu, H. (2018). ALS mutations of FUS suppress protein translation and disrupt the regulation of nonsense-mediated decay. Proc. Natl. Acad. Sci. U.S.A. 115, E11904-E11913.

Kanekura, K., Yagi, T., Cammack, A. J., Mahadevan, J., Kuroda, M., Harms, M. B., et al. (2016). Poly-dipeptides encoded by the C9ORF72 repeats block global protein translation. Hum. Mol. Genet. 25, 1803-1813. doi: 10.1093/hmg/ ddw052

Kino, Y., Washizu, C., Kurosawa, M., Yamada, M., Miyazaki, H., Akagi, T., et al. (2015). FUS/TLS deficiency causes behavioral and pathological abnormalities distinct from amyotrophic lateral sclerosis. Acta Neuropathol. Commun. 3:24.

Kitamura, A., Nakayama, Y., Shibasaki, A., Taki, A., Yuno, S., Takeda, K., et al. (2016). Interaction of RNA with a C-terminal fragment of the amyotrophic lateral sclerosis-associated TDP43 reduces cytotoxicity. Sci. Rep. 6:19230.

Klim, J. R., Williams, L. A., Limone, F., Guerra San Juan, I., Davis-Dusenbery, B. N., Mordes, D. A., et al. (2019). ALS-implicated protein TDP-43 sustains levels of STMN2, a mediator of motor neuron growth and repair. Nat. Neurosci. 22, 167-179. doi: 10.1038/s41593-018-0300-4

Kramer, N. J., Haney, M. S., Morgens, D. W., Jovicic, A., Couthouis, J., Li, A., et al. (2018). CRISPR-Cas9 screens in human cells and primary neurons identify modifiers of C9ORF72 dipeptide-repeat-protein toxicity. Nat. Genet. 50, 603-612. doi: 10.1038/s41588-018-0070-7

Kuo, P. H., Chiang, C. H., Wang, Y. T., Doudeva, L. G., and Yuan, H. S. (2014). The crystal structure of TDP-43 RRM1-DNA complex reveals the specific recognition for UG- and TG-rich nucleic acids. Nucleic Acids Res. 42, 47124722. doi: 10.1093/nar/gkt1407

Kurosaki, T., and Maquat, L. E. (2016). Nonsense-mediated mRNA decay in humans at a glance. J. Cell Sci. 129, 461-467.

Kwiatkowski, T. J. Jr., Bosco, D. A., Leclerc, A. L., Tamrazian, E., Vanderburg, C. R., Russ, C., et al. (2009). Mutations in the FUS/TLS gene on chromosome 16 cause familial amyotrophic lateral sclerosis. Science 323, 1205-1208.

Lagier-Tourenne, C., Baughn, M., Rigo, F., Sun, S., Liu, P., Li, H. R., et al. (2013). Targeted degradation of sense and antisense C9orf72 RNA foci as therapy for ALS and frontotemporal degeneration. Proc. Natl. Acad. Sci. U.S.A. 110, E4530-E4539.
Lee, Y. B., Chen, H. J., Peres, J. N., Gomez-Deza, J., Attig, J., Stalekar, M., et al. (2013). Hexanucleotide repeats in ALS/FTD form length-dependent RNA foci, sequester RNA binding proteins, and are neurotoxic. Cell Rep. 5, 1178-1186. doi: 10.1016/j.celrep.2013.10.049

Li, W., Reeb, A. N., Lin, B., Subramanian, P., Fey, E. E., Knoverek, C. R., et al. (2017). Heat shock-induced phosphorylation of TAR DNA-binding protein 43 (TDP-43) by MAPK/ERK kinase regulates TDP-43 function. J. Biol. Chem. 292, 5089-5100. doi: 10.1074/jbc.m116.753913

Lim, A., Rechtsteiner, A., and Saxton, W. M. (2017). Two kinesins drive anterograde neuropeptide transport. Mol .Biol. Cell 28, 3542-3553. doi: 10. 1091/mbc.e16-12-0820

Ling, S. C., Polymenidou, M., and Cleveland, D. W. (2013). Converging mechanisms in ALS and FTD: disrupted RNA and protein homeostasis. Neuron 79, 416-438. doi: 10.1016/j.neuron.2013.07.033

Liu, J., Hu, J., Ludlow, A. T., Pham, J. T., Shay, J. W., Rothstein, J. D., et al. (2017). c9orf72 disease-related foci are each composed of one mutant expanded repeat RNA. Cell Chem. Biol. 24, 141-148. doi: 10.1016/j.chembiol.2016.12.018

Liu, X., Niu, C., Ren, J., Zhang, J., Xie, X., Zhu, H., et al. (2013). The RRM domain of human fused in sarcoma protein reveals a noncanonical nucleic acid binding site. Biochim. Biophys. Acta 1832, 375-385. doi: 10.1016/j.bbadis.2012.11.012

Liu-Yesucevitz, L., Lin, A. Y., Ebata, A., Boon, J. Y., Reid, W., Xu, Y. F., et al. (2014). ALS-linked mutations enlarge TDP-43-enriched neuronal RNA granules in the dendritic arbor. J. Neurosci. 34, 4167-4174. doi: 10.1523/jneurosci.2350-13. 2014

Lopez-Erauskin, J., Tadokoro, T., Baughn, M. W., Myers, B., McAlonis-Downes, M., Chillon-Marinas, C., et al. (2018). ALS/FTD-linked mutation in fus suppresses intra-axonal protein synthesis and drives disease without nuclear loss-of-function of FUS. Neuron 100, 816-830 e7.

Loughlin, F. E., Lukavsky, P. J., Kazeeva, T., Reber, S., Hock, E. M., Colombo, M., et al. (2019). The solution structure of FUS bound to RNA reveals a bipartite mode of RNA recognition with both sequence and shape specificity. Mol. Cell 73, 490-504 e6.

Lu, Y., Lim, L., Song, J., and domain, R. R. M. (2017). of ALS/FTD-causing FUS characteristic of irreversible unfolding spontaneously self-assembles into amyloid fibrils. Sci. Rep. 7:1043.

Ly, C. V., and Miller, T. M. (2018). Emerging antisense oligonucleotide and viral therapies for amyotrophic lateral sclerosis. Curr. Opin. Neurol. 31, 648-654. doi: 10.1097/wco.0000000000000594

Lykke-Andersen, J., Shu, M. D., and Steitz, J. A. (2000). Human Upf proteins target an mRNA for nonsense-mediated decay when bound downstream of a termination codon. Cell 103, 1121-1131. doi: 10.1016/s0092-8674(00)00214-2

Mackness, B. C., Tran, M. T., McClain, S. P., Matthews, C. R., and Zitzewitz, J. A. (2014). Folding of the RNA recognition motif (RRM) domains of the amyotrophic lateral sclerosis (ALS)-linked protein TDP-43 reveals an intermediate state. J. Biol. Chem. 289, 8264-8276. doi: 10.1074/jbc.m113. 542779

Maharana, S., Wang, J., Papadopoulos, D. K., Richter, D., Pozniakovsky, A., Poser, I., et al. (2018). RNA buffers the phase separation behavior of prion-like RNA binding proteins. Science 360, 918-921. doi: 10.1126/science.aar7366

Mann, J. R., Gleixner, A. M., Mauna, J. C., Gomes, E., DeChellis-Marks, M. R., Needham, P. G., et al. (2019). Neurotoxic phase transitions of TDP-43. Neuron 102, 321-338 e8.

Melamed, Z., Lopez-Erauskin, J., Baughn, M. W., Zhang, O., Drenner, K., Sun, Y., et al. (2019). Premature polyadenylation-mediated loss of stathmin-2 is a hallmark of TDP-43-dependent neurodegeneration. Nat. Neurosci. 22, 180-190. doi: 10.1038/s41593-018-0293-z

Mitra, J., Guerrero, E. N., Hegde, P. M., Liachko, N. F., Wang, H., Vasquez, V., et al. (2019). Motor neuron disease-associated loss of nuclear TDP-43 is linked to DNA double-strand break repair defects. Proc. Natl. Acad. Sci. U.S.A. 116, 4696-4705. doi: 10.1073/pnas.1818415116

Mizielinska, S., Gronke, S., Niccoli, T., Ridler, C. E., Clayton, E. L., Devoy, A., et al. (2014). C9orf72 repeat expansions cause neurodegeneration in Drosophila through arginine-rich proteins. Science 345, 1192-1194. doi: 10.1126/science. 1256800

Mizielinska, S., Ridler, C. E., Balendra, R., Thoeng, A., Woodling, N. S., Grasser, F. A., et al. (2017). Bidirectional nucleolar dysfunction in C9orf72 frontotemporal lobar degeneration. Acta Neuropathol. Commun. 5:29. 
Monahan, Z., Ryan, V. H., Janke, A. M., Burke, K. A., Rhoads, S. N., Zerze, G. H., et al. (2017). Phosphorylation of the FUS low-complexity domain disrupts phase separation, aggregation, and toxicity. EMBO J. 36, 2951-2967. doi: 10. 15252/embj. 201696394

Mulholland, K., Sullivan, H. J., Garner, J., Cai, J., Chen, B., and Wu, C. (2020). Three-dimensional structure of RNA monomeric G-quadruplex containing ALS and FTD related G4C2 repeat and its binding with TMPyP4 probed by homology modeling based on experimental constraints and molecular dynamics simulations. ACS Chem. Neurosci. 11, 57-75. doi: 10.1021/ acschemneuro.9b00572

Nakaya, T., Alexiou, P., Maragkakis, M., Chang, A., and Mourelatos, Z. (2013). FUS regulates genes coding for RNA-binding proteins in neurons by binding to their highly conserved introns. RNA 19, 498-509. doi: 10.1261/rna.037804.112

Nana, A. L., Sidhu, M., Gaus, S. E., Hwang, J. L., Li, L., Park, Y., et al. (2019). Neurons selectively targeted in frontotemporal dementia reveal early stage TDP-43 pathobiology. Acta Neuropathol. 137, 27-46. doi: 10.1007/s00401-0181942-8

Neelagandan, N., Gonnella, G., Dang, S., Janiesch, P. C., Miller, K. K., Kuchler, K., et al. (2019). TDP-43 enhances translation of specific mRNAs linked to neurodegenerative disease. Nucleic Acids Res. 47, 341-361. doi: 10.1093/nar/ gky972

Neumann, M., Sampathu, D. M., Kwong, L. K., Truax, A. C., Micsenyi, M. C., Chou, T. T., et al. (2006). Ubiquitinated TDP-43 in frontotemporal lobar degeneration and amyotrophic lateral sclerosis. Science 314, 130-133.

Niaki, A. G., Sarkar, J., Cai, X., Rhine, K., Vidaurre, V., Guy, B., et al. (2020). Loss of dynamic RNA interaction and aberrant phase separation induced by two distinct types of ALS/FTD-linked FUS mutations. Mol. Cell 77, 82-94.e4.

Nicolas, A., Kenna, K. P., Renton, A. E., Ticozzi, N., Faghri, F., Chia, R., et al. (2018). Genome-wide analyses identify KIF5A as a novel ALS gene. Neuron 97, $1268-1283$ e6.

Ortega, J. A., Daley, E. L., Kour, S., Samani, M., Tellez, L., Smith, H. S., et al. (2020). Nucleocytoplasmic proteomic analysis uncovers eRF1 and nonsense-mediated decay as modifiers of ALS/FTD C9orf72 toxicity. Neuron 106, 90-107 e13.

Ou, S. H., Wu, F., Harrich, D., Garcia-Martinez, L. F., and Gaynor, R. B. (1995). Cloning and characterization of a novel cellular protein, TDP-43, that binds to human immunodeficiency virus type 1 TAR DNA sequence motifs. J. Virol. 69, 3584-3596. doi: 10.1128/jvi.69.6.3584-3596.1995

Pesiridis, G. S., Lee, V. M. Y., and Trojanowski, J. Q. (2009). Mutations in TDP-43 link glycine-rich domain functions to amyotrophic lateral sclerosis. Hum. Mol. Genet. 18, R156-R162.

Reber, S., Mechtersheimer, J., Nasif, S., Benitez, J. A., Colombo, M., Domanski, M., et al. (2018). A clean approach for the generation of gene knockouts and gene replacements in human cells. Mol. Biol. Cell 29, 75-83. doi: 10.1091/mbc.e1705-0288

Reddy, K., Zamiri, B., Stanley, S. Y., Macgregor, R. B. Jr., and Pearson, C. E. (2013). The disease-associated r(GGGGCC)n repeat from the C9orf72 gene forms tract length-dependent uni- and multimolecular RNA G-quadruplex structures. J. Biol. Chem. 288, 9860-9866. doi: 10.1074/jbc.c113.452532

Renton, A. E., Majounie, E., Waite, A., Simon-Sanchez, J., Rollinson, S., Gibbs, J. R., et al. (2011). A hexanucleotide repeat expansion in C9ORF72 is the cause of chromosome 9p21-linked ALS-FTD. Neuron 72, 257-268.

Robinson, H. K., Deykin, A. V., Bronovitsky, E. V., Ovchinnikov, R. K., Ustyugov, A. A., Shelkovnikova, T. A., et al. (2015). Early lethality and neuronal proteinopathy in mice expressing cytoplasm-targeted FUS that lacks the RNA recognition motif. Amyotroph. Lateral Scler. Frontotemporal Degener. 16, 402409. doi: 10.3109/21678421.2015.1040994

Romano, M., Feiguin, F., and Buratti, E. (2016). TBPH/TDP-43 modulates translation of Drosophila futsch mRNA through an UG-rich sequence within its 5'UTR. Brain Res. 1647, 50-56. doi: 10.1016/j.brainres. 2016.02.022

Russo, A., Scardigli, R., La Regina, F., Murray, M. E., Romano, N., Dickson, D. W., et al. (2017). Increased cytoplasmic TDP-43 reduces global protein synthesis by interacting with RACK1 on polyribosomes. Hum. Mol. Genet. 26, 1407-1418. doi: $10.1093 / \mathrm{hmg} / \mathrm{ddx} 035$

Shang, Y., and Huang, E. J. (2016). Mechanisms of FUS mutations in familial amyotrophic lateral sclerosis. Brain Res. 1647, 65-78. doi: 10.1016/j.brainres. 2016.03.036
Shi, Y., Lin, S., Staats, K. A., Li, Y., Chang, W. H., Hung, S. T., et al. (2018). Haploinsufficiency leads to neurodegeneration in C9ORF72 ALS/FTD human induced motor neurons. Nat. Med. 24, 313-325.

Shodai, A., Ido, A., Fujiwara, N., Ayaki, T., Morimura, T., Oono, M., et al. (2012). Conserved acidic amino acid residues in a second RNA recognition motif regulate assembly and function of TDP-43. PLoS One 7:e52776. doi: 10.1371/ journal.pone.0052776

Shodai, A., Morimura, T., Ido, A., Uchida, T., Ayaki, T., Takahashi, R., et al. (2013). Aberrant assembly of RNA recognition motif 1 links to pathogenic conversion of TAR DNA-binding protein of $43 \mathrm{kDa}$ (TDP-43). J. Biol. Chem. 288, 14886-14905. doi: 10.1074/jbc.m113.451849

Sreedharan, J., Blair, I. P., Tripathi, V. B., Hu, X., Vance, C., Rogelj, B., et al. (2008). TDP-43 mutations in familial and sporadic amyotrophic lateral sclerosis. Science 319, 1668-1672.

Sun, Y., Arslan, P. E., Won, A., Yip, C. M., and Chakrabartty, A. (2014). Binding of TDP-43 to the 3'UTR of its cognate mRNA enhances its solubility. Biochemistry 53, 5885-5894. doi: 10.1021/bi500617x

Sun, Y., Eshov, A., Zhou, J., Isiktas, A. U., and Guo, J. U. (2020). C9orf72 argininerich dipeptide repeats inhibit UPF1-mediated RNA decay via translational repression. Nat. Commun. 11:3354.

Tabet, R., Schaeffer, L., Freyermuth, F., Jambeau, M., Workman, M., Lee, C. Z., et al. (2018). CUG initiation and frameshifting enable production of dipeptide repeat proteins from ALS/FTD C9ORF72 transcripts. Nat. Commun. 9:152.

Takanashi, K., and Yamaguchi, A. (2014). Aggregation of ALS-linked FUS mutant sequesters RNA binding proteins and impairs RNA granules formation. Biochem. Biophys. Res. Commun. 452, 600-607. doi: 10.1016/j.bbrc.2014.08.115

Tank, E. M., Figueroa-Romero, C., Hinder, L. M., Bedi, K., Archbold, H. C., and Li, X. (2018). Abnormal RNA stability in amyotrophic lateral sclerosis. Nat. Commun. 9:2845.

Tao, Z., Wang, H., Xia, Q., Li, K., Li, K., Jiang, X., et al. (2015). Nucleolar stress and impaired stress granule formation contribute to C9orf72 RAN translationinduced cytotoxicity. Hum. Mol. Genet. 24, 2426-2441. doi: 10.1093/hmg/ ddv005

Tollervey, J. R., Curk, T., Rogelj, B., Briese, M., Cereda, M., Kayikci, M., et al. (2011). Characterizing the RNA targets and position-dependent splicing regulation by TDP-43. Nat. Neurosci. 14, 452-458. doi: 10.1038/nn.2778

Tran, H., Almeida, S., Moore, J., Gendron, T. F., Chalasani, U., Lu, Y., et al. (2015). Differential toxicity of nuclear RNA Foci versus dipeptide repeat proteins in a Drosophila Model of C9ORF72 FTD/ALS. Neuron 87, 1207-1214. doi: 10.1016/ j.neuron.2015.09.015

Vance, C., Rogelj, B., Hortobagyi, T., De Vos, K. J., Nishimura, A. L., Sreedharan, J., et al. (2009). Mutations in FUS, an RNA processing protein, cause familial amyotrophic lateral sclerosis type 6. Science 323, 1208-1211.

Vivoli Vega, M., Nigro, A., Luti, S., Capitini, C., Fani, G., Gonnelli, L., et al. (2019). Isolation and characterization of soluble human full-length TDP-43 associated with neurodegeneration. FASEB J. 33, 10780-10793. doi: 10.1096/fj.201900474r

Voigt, A., Herholz, D., Fiesel, F. C., Kaur, K., Muller, D., Karsten, P., et al. (2010). TDP-43-mediated neuron loss in vivo requires RNA-binding activity. PLoS One 5:e12247. doi: 10.1371/journal.pone.0012247

Waite, A. J., Baumer, D., East, S., Neal, J., Morris, H. R., Ansorge, O., et al. (2014). Reduced C9orf72 protein levels in frontal cortex of amyotrophic lateral sclerosis and frontotemporal degeneration brain with the C9ORF72 hexanucleotide repeat expansion. Neurobiol. Aging 35, 1779 e5-1779 e13.

Webster, C. P., Smith, E. F., Grierson, A. J., and De Vos, K. J. (2018). C9orf72 plays a central role in rab GTPase-dependent regulation of autophagy. Small GTPases 9, 399-408. doi: 10.1080/21541248.2016.1240495

Weskamp, K., Tank, E. M., Miguez, R., McBride, J. P., Gomez, N. B., White, M., et al. (2020). Shortened TDP43 isoforms upregulated by neuronal hyperactivity drive TDP43 pathology in ALS. J. Clin. Invest. 130, 1139-1155. doi: 10.1172/ jci130988

Xiao, S., Sanelli, T., Dib, S., Sheps, D., Findlater, J., Bilbao, J., et al. (2011). RNA targets of TDP-43 identified by UV-CLIP are deregulated in ALS. Mol. Cell Neurosci. 47, 167-180. doi: 10.1016/j.mcn.2011.02.013

Xu, W., Bao, P., Jiang, X., Wang, H., Qin, M., Wang, R., et al. (2019). Reactivation of nonsense-mediated mRNA decay protects against C9orf72 dipeptide-repeat neurotoxicity. Brain 142, 1349-1364. doi: 10.1093/brain/awz070

Yang, K., Yang, J., and Yi, J. (2018). Nucleolar Stress: hallmarks, sensing mechanism and diseases. Cell Stress 2, 125-140. doi: 10.15698/cst2018.06.139 
Yasuda, K., Clatterbuck-Soper, S. F., Jackrel, M. E., Shorter, J., and Mili, S. (2017). FUS inclusions disrupt RNA localization by sequestering kinesin-1 and inhibiting microtubule detyrosination. J. Cell Biol. 216, 1015-1034. doi: $10.1083 /$ jcb.201608022

Zacco, E., Grana-Montes, R., Martin, S. R., de Groot, N. S., Alfano, C., Tartaglia, G. G., et al. (2019). RNA as a key factor in driving or preventing self-assembly of the TAR DNA-binding protein 43. J. Mol. Biol. 431, 1671-1688. doi: 10.1016/ j.jmb.2019.01.028

Zaepfel, B. L., Zhang, Z., Maulding, K., Coyne, A. N., Cheng, W., Hayes, L. R., et al. (2021). UPF1 reduces C9orf72 HRE-induced neurotoxicity in the absence of nonsense-mediated decay dysfunction. Cell Rep. 34:108925. doi: 10.1016/j. celrep.2021.108925

Zhang, K., Donnelly, C. J., Haeusler, A. R., Grima, J. C., Machamer, J. B., Steinwald, P., et al. (2015). The C9orf72 repeat expansion disrupts nucleocytoplasmic transport. Nature 525, 56-61. doi: 10.1038/nature14973

Zhang, Y. J., Gendron, T. F., Ebbert, M. T. W., O’Raw, A. D., Yue, M., JansenWest, K., et al. (2018). Poly(GR) impairs protein translation and stress granule dynamics in C9orf72-associated frontotemporal dementia and amyotrophic lateral sclerosis. Nat. Med. 24, 1136-1142. doi: 10.1038/s41591-018-0071-1

Zu, T., Liu, Y., Banez-Coronel, M., Reid, T., Pletnikova, O., Lewis, J., et al. (2013). RAN proteins and RNA foci from antisense transcripts in C9ORF72 ALS and frontotemporal dementia. Proc. Natl. Acad. Sci. U.S.A. 110, E4968-E4977.

Conflict of Interest: The authors declare that the research was conducted in the absence of any commercial or financial relationships that could be construed as a potential conflict of interest.

Copyright (C) 2021 Zaepfel and Rothstein. This is an open-access article distributed under the terms of the Creative Commons Attribution License (CC BY). The use, distribution or reproduction in other forums is permitted, provided the original author(s) and the copyright owner(s) are credited and that the original publication in this journal is cited, in accordance with accepted academic practice. No use, distribution or reproduction is permitted which does not comply with these terms. 